\title{
Féeries
}

Études sur le conte merveilleux, XVII ${ }^{\mathrm{e}} \mathrm{XIX}{ }^{\mathrm{e}}$ siècle

4 | 2007

Le conte, la scène

\section{Cendrillon mise en pièces ou la seconde immortalité de Perrault au XIX ${ }^{\mathrm{e}}$ siècle}

\section{Noémie Courtès}

\section{(2) OpenEdition}

Journals

Édition électronique

URL : http://journals.openedition.org/feeries/273

DOI : $10.4000 /$ feeries. 273

ISSN : $1957-7753$

\section{Éditeur}

UGA Éditions/Université Grenoble Alpes

\section{Édition imprimée}

Date de publication : 1 octobre 2007

Pagination : 73-88

ISBN : 978-2-84310-101-4

ISSN : 1766-2842

\section{Référence électronique}

Noémie Courtès, "Cendrillon mise en pièces ou la seconde immortalité de Perrault au xix ${ }^{\mathrm{e}}$ siècle ", Féeries [En ligne], 4 | 2007, mis en ligne le 08 décembre 2011, consulté le 08 septembre 2020. URL: http://journals.openedition.org/feeries/273 ; DOI : https://doi.org/10.4000/feeries.273

Ce document a été généré automatiquement le 8 septembre 2020.

(c) Féeries 


\title{
Cendrillon mise en pièces ou la seconde immortalité de Perrault au XIX ${ }^{\mathrm{e}}$ siècle
}

\author{
Noémie Courtès
}

1 LA SCÈNE S'EST TRÈS TÔT EMPARÉE DES INTRIGUES DES CONTES DE FÉES, que ce soit en adaptant telles quelles les intrigues, ou en en utilisant seulement les procédés, sur les théâtres officiels comme à la Foire et à l'Opéra ${ }^{1}$, ou encore sur des scènes privées ${ }^{2}$. Et si la continuité fut assurée dans la littérature narrative entre les premiers recueils de contes (de $\mathrm{M}^{\mathrm{me}}$ d'Aulnoy et de Perrault) et la perversion ${ }^{3}$ du féerique « fin de siècle » à la Banville, par le biais des simplifications pour la jeunesse ou l'apport de l'étranger (les frères Grimm ou Andersen entre autres), le féerique connut une autre efflorescence pendant la période romantique et jusqu'à la veille de la première guerre mondiale - lorsque l'invention du cinéma prit le relais - dans le domaine dramatique.

2 Après la Révolution en effet, le public se modifie dans le sens d'une extension sans précédent, et lorsque les auteurs dédaignent la pression de l'actualité (le théâtre révolutionnaire) ou l'attraction de l'Histoire (le drame romantique), ils se détournent aussi des mythes classiques pour aller chercher des canevas plus immédiatement compréhensibles et des effets plus faciles - l'aboutissement de ce mouvement étant l' Orphée aux Enfers d'Offenbach, qui passe de deux actes et quatre tableaux en 1858 à quatre actes et douze tableaux en 1874 pour en augmenter le spectaculaire. Entre temps, cette tendance a touché nombre de genres scéniques - en constante redéfinition en fonction des libertés ou de la censure, révolutionnaires, impériales ou royales, qui s'appliquent au théâtre : dans le ballet par exemple, l'invention des « pointes » dans les années 1820 permet de donner l'impression que la ballerine vole, ce qui autorise la création de plusieurs ballets utilisant des êtres elfiques (La Sylphide, Giselle, La Péri), à moins que ce ne soit la volonté d'employer de tels personnages qui ait abouti à cette innovation.

3 Le féerique prend tant d'importance qu'un genre est même crée à sa dévotion: la «féerie ${ }^{4}$ ». À la fois catégorie esthétique - du Directoire à la Monarchie de Juillet, 
lorsque l'expérimentation et la liberté des théâtres permettent une extrême porosité des techniques d'écriture - et genre à part entière - après 1840, lorsque le rétablissement de la censure et des privilèges des théâtres formalise le schéma de construction des intrigues qui finira par se scléroser -, la féerie dramatique comprend plusieurs centaines de pièces et est révélatrice des tensions à l'œuvre dans les genres limitrophes (vaudeville, pantomime, ballet, opéra) pour dépasser l'illusion théâtrale et entrer dans l'illusion féerique ${ }^{5}$.

4 Vouloir entreprendre en quelques pages une synthèse de ce mouvement de fond des arts de la scène au XIXe siècle serait illusoire. Cette contribution se veut donc une simple illustration, à partir de l'exemple de Cendrillon, de la diversité des variations sur ce thème, de l'esprit féerique à la scène et des efforts mis en œuvre pour satisfaire un public avide de divertissements oniriques.

\section{Cendrillon ou comment trouver pantoufle à son pied}

Les Contes de Ma Mère l'Oye de Perrault ont connu un engouement sans discontinuité depuis 1697 jusqu'à la fin du XIX ${ }^{e}$ siècle ${ }^{6}$, des Fées ou les Contes de Ma Mère l'oie, Comédie en un acte Mise au théâtre par Messieurs du $\mathrm{F}^{* *} \& \mathrm{~B}^{* * * *}$, \& représentée pour la première fois par les Comédiens italiens du Roi dans leur Hôtel de Bourgogne, le $2^{\mathrm{e}}$ jour de mars 1697, au film de Méliès tourné en 1899, Cendrillon, en passant par Les Contes de la Mère l'Oie, grande féerie en cinq actes et vingt-deux tableaux par MM. Clairville et Jules Cordier, représentée pour la première fois, à Paris, sur le théâtre de l'Ambigu-Comique, le 20 mai 1854.

Le résultat est parfois effarant: dans ce dernier exemple, la "grande féerie " de Clairville et Cordier, tous les contes sont hachés menus et mélangés au gré d'une bonne fée (mère l'Oie) et d'une mauvaise (Serpentine), alternativement sous forme humaine et sous la forme de l'animal qui leur donne son nom, qui favorisent ou empêchent l'hymen de Simplette avec Riquet à la houppe, opportunément retransformé en Prince charmant à la fin. Le tout à grand renfort d'ogres croqueurs de grand-mères et de pantoufles enchantées, d'allusions au théâtre contemporain et de calembours effroyables.

7 Les contemporains n'hésitent pas à ironiser sur cet engouement extraordinaire des arts de la scène pour Perrault :

C'est curieux de voir tout ce que Charles Perrault [...] a fourni au théâtre. [...] Depuis on en a fait des opéras, des ballets, des féeries, des vaudevilles et si Perrault, renouvelant l'exemple d'Abraham et de Mathusalem, vivait encore, il aurait réalisé une jolie fortune avec sa part obligée de collaborateur. Ses droits d'auteur auraient certainement atteint plusieurs centaines de mille francs. ${ }^{?}$

8 Ainsi, Perrault, « immortel » depuis son élection par ses pairs à l'Académie française en 1671, a-t-il été élevé une seconde fois à l'immortalité, par le plébiscite du public et ses réécritures successives cette fois, entre 1810 et $1899^{8}$.

9 Mais si la plupart des contes de Perrault ont connu des adaptations ${ }^{9}$, c'est incontestablement Cendrillon qui est le meilleur exemple de l'engouement pour l'esprit des contes. En effet, plus de trente ${ }^{10}$ Cendrillon ont vu le jour au XIX ${ }^{e}$ siècle, dans des genres différents ou hybrides ${ }^{11}$. 
10 La première Cendrillon du siècle date de $1806^{12}$, mais la véritable vogue est lancée en 1810 par l'opéra-féerie du même nom d'Étienne et Nicolo $^{13}$. Le succès est immédiat (une centaine de représentations successives) et en l'espace d'une année, onze spectacles (dont un représenté à Montpellier, Les Pantoufles de Magdelaine, et un autre publié à Lyon, Le Mariage de Cendrillon) lui font suite. La composante parodique est primordiale dans cette série, mais même lorsqu'il n'y a pas d'intention parodique ou qu'il y a transgénérisme, un jeu d'intertextualité intense est à l'œuvre dans ces pièces: la chanson « compère Guilleri » passe par exemple du ballet de $1809^{14}$ à l'œuvre d'Étienne en 1810, puis est repris d'une façon ou d'une autre dans les parodies et même les pièces suivantes (parfois pour un vaudeville, parfois dans le nom d'un personnage, etc.). Entre temps, le texte d'Étienne a influencé les autres pendant plus de deux décennies et s'est adapté à tous les genres, puisque l'intrigue du ballet de 1823 et le vaudeville-féerie de 1838 s'en inspirent étroitement, ainsi que l'opéra de Rossini, La Cenerentola (Cendrillon ou le triomphe de la bonté dans la version française), de 1817, qui en reprend l'intrigue point par point. La gloire de la pièce d'Étienne est ensuite assurée par les reprises de 1845 et 1877 , dans des mises en scène étendues et encore plus brillantes.

11 En 1810, les pièces tiennent à chaque fois compte de toutes les précédentes et les différentes versions jouent de l'empilement de ces références, augmentant ainsi progressivement le nombre des Cendrillons : quatre dans La Famille des Cendrillons, cinq dans La Fête de Perrault, six dans Le Rendez-vous des Cendrillons. L'effet comique culmine à la fin du Rendez-vous des Cendrillons, lorsqu'un commis arrive, annonçant trois nouvelles Cendrillons (deux du Boulevard, une d'Italie) si bien qu'on fait fermer les portes pour endiguer l'invasion... Déjà, dans La Famille des Cendrillons, les quatre Cendrillons essayaient en vain la pantoufle parce qu'elle était - au contraire de celle du conte de Perrault - trop grande: "Tant de gens ont essayé cette pantoufle, qu'il n'est pas étonnant qu'elle soit élargie ». Et le vaudeville final insistait sur cette intrigue à tout faire :

Sur la vogue des Cendrillons

Tout le monde, aujourd'hui, spécule ;

Aussi pièces, chapeaux, bonbons,

Usurpent ce nom sans scrupule, [...]. (sc. 13 et finale)

12 L'intérêt des réécritures venait également de l'adaptation aux contraintes particulières à chaque théâtre et à la censure : la version de l'odéon est une pure comédie, celle de Séraphin emploie des marionnettes, d'autres s'intéressent davantage aux possibilités du vaudeville, etc. En 1810 en effet, chaque théâtre était spécialisé par ordonnance impériale et devait ruser s'il voulait ne serait-ce qu'empiéter sur le domaine de ses concurrents directs.

13 Ensuite, les versions s'espacent, mais continuent à occuper toutes les scènes. Même une institution comme l'Académie royale de musique reprend le thème, avec le ballet d'Albert Decombe en 1823. Dans des décors de Ciceri, ce qui montre bien le continuum entre opéra, ballet et théâtre pour le spectateur du XIXe siècle.

14 Ce qui frappe aujourd'hui, c'est la diversité de ces versions, alors qu'elles sont construites autour d'un même sujet. Les modifications touchent tous les éléments possibles: le lieu (de Bordeaux à la Turquie en passant par l'Italie), le temps (du Moyen-Âge au XVI ${ }^{e}$ siècle ou à l'« époque Louis XIII »), le nombre des personnages (une marâtre et deux sœurs ; deux sœurs ; un parâtre et une sœur ou deux), l'onomastique (riche et signifiante : la comtesse de Hautainville, Uranie de La Houspignole, $\mathrm{M}^{\mathrm{me}}$ de La 
Haltière, ...), le statut du prince (sultan, roi ou prince-enfant), l'identité de la fée (génie, Grand Astrologue ou chatte blanche dite "fée Minette »), l'heure limite (de minuit à deux heures du matin) et jusqu'à la pantoufle (soulier vert ou de verre) qui peut être remplacée par un bracelet ou une rose... Même la magie peut s'effacer, comme dans le texte d'Étienne, qui substitue à la fée un précepteur avisé, Alidor - «dont on raconte des choses fort extraordinaires : il sait toutes les langues, il lit dans les astres; on dit même qu'il est en intelligence avec des génies » $(\mathrm{I}, 3)$ - et ne justifie pas le pouvoir du talisman donné à l'héroïne ; il utilise cependant un " chœur aérien qui est censé chanté par des génies » au deuxième acte (sc. 1) et réintroduit une fée dans le divertissement final pour faire bonne mesure. Excepté le livret mis en musique par Rossini (très fidèle), l'absence de magie est le seul élément que les textes postérieurs influencés par Étienne ne conservent pas, au contraire, puisqu'ils soulignent volontiers le caractère surnaturel de l'histoire.

Le centre de gravité peut en outre être déplacé, en particulier par l'adjonction de personnages ou d'épisodes exogènes : déjà Étienne mettait au premier plan la ruse très marivaudienne d'avoir échangé l'identité du prince Ramir avec un seigneur ridicule (Dandini) pour éprouver le désintéressement de Cendrillon, et la version de Massenet ${ }^{15}$ inclut une scène très poétique de prières à la fée, simultanément chantées par Cendrillon et le Prince Charmant de part et d'autre d'un mur de fleurs magiquement apparu pour les séparer sur le modèle de Pyrame et Thisbé (III, ${ }^{\mathrm{er}}$ tableau, sc. 4).

Il faut donc en conclure que le « meccano du conte ${ }^{16} »$ joue à plein également dans la sphère dramatique.

\section{Les ingrédients du féerique à la scène}

Comme le remarque Pierre Vidal ${ }^{17}$, l'histoire de Cendrillon a tous les atouts pour faire une bonne adaptation puisque, outre une quasi unité de lieu et de temps (par rapport à La Belle au bois dormant), l'héroïne est touchante et le sujet non pervers (à la différence de Peau d'Âne). Le passage à la scène au $\mathrm{XIX}^{\mathrm{e}}$ siècle va cependant ajouter de nouveaux attraits au conte, même si, contrairement aux adaptations narratives de la fin du siècle, on ne note pas d'actualisation obligatoire, ni d'implication outrancièrement érotique, ni de considérations polémiques sur la condition de la femme ${ }^{18}$.

Parce que le seul objectif est de séduire et d'émouvoir, la scène respecte assez fidèlement la situation déplorable de Cendrillon, et s'attache à jouer sur toutes les variantes possibles, qu'elles soient génériques ou techniques. Car le succès des Cendrillon s'étend sur un siècle, et un siècle particulièrement riche en expérimentations théâtrales comme en inventions scénographiques (lumières et effets d'optique, utilisation de l'électricité et de la pyrotechnie, truquages et trappes anglaises, etc.). Si bien que l'ampleur des variations défie la synthèse, tant le phénomène, entre parodies et féeries, ballets et opéras, se révèle complexe. Il semble cependant que l'on puisse relever au moins trois facteurs essentiels dans le succès perraldien au XIX ${ }^{e}$ siècle : le comique, la musique et la primauté du spectaculaire.

19 À la différence du conte, qui reste sérieux de bout en bout ${ }^{19}$, ces adaptations dramatiques contiennent toutes un élément de comique. Plus ou moins appuyé, il tient du burlesque dans les parodies, qui, outre l'ironie sur l'hypotexte, peuvent user d'une héroïne interprétée par un homme: dans La Chatte merveilleuse, les spectateurs 
pouvaient reconnaître dans le rôle de Cendrillon le comédien Brunet, resté célèbre pour son rôle de " Jocrisse ». Dans d'autres œuvres, c'est la symétrie des deux sœurs qui est exploitée (en particulier dans les ballets), ou encore l'ajout d'un rival grotesque (Guilleri, Dandini ou Bambini, etc.) à la trame de base, procédé qui s'ajoute par exemple au précédent chez Étienne, lequel calque une scène de séduction entre Dandini, Clorinde et Tisbé (les sœurs de Cendrillon) sur la scène de séduction des deux paysannes dans le Dom Juan de Molière ${ }^{20}$. Le tout est de contrebalancer ponctuellement les malheurs de Cendrillon.

Quant à la musique, elle est très présente car le conte peut être un prétexte facile à son emploi : non seulement les auteurs peuvent compter sur l'épisode des sonnettes (lorsque ses sœurs martyrisent Cendrillon en l'appelant aux deux bouts d'un appartement central - sur le modèle de la première scène du Mariage de Figaro), mais en outre le bal est l'occasion rêvée d'insérer des scènes de danse, ce dont les ballets et les opéras ne se privent évidemment pas (Henri Cain ménage ainsi cinq entrées différentes dans le bal qui constitue le deuxième acte de l'opéra-comique de Massenet). L'atmosphère onirique de ces spectacles permet également de privilégier et de mettre en valeur les voix de femmes, en multipliant les vocalises chez Rossini, en faisant intervenir des chœurs d'esprits et en faisant interpréter le Prince Charmant par une «soprano de sentiment» chez Massenet. Quant aux pièces parlées, en accord avec l'esthétique et la mode du XIX ${ }^{\mathrm{e}}$ siècle, elles exploitent presque toutes les ressources du vaudeville, qui y est récurrent et renforce souvent le comique, l'emploi d'un air connu ajoutant en effet du sens aux couplets chantés : dans La Chatte merveilleuse, le départ de Cendrillon pour le bal se fait sur l'air de «Bon voyage monsieur Dumollet ", et à la fin de la Fête de Perrault, Cendrillon danse pour Barbe-bleue, le Petit-Poucet, la Belle-au-bois-dormant (etc.) sur l'air «Où peut-on être mieux qu'au sein de sa famille $» . .$.

21 Mais si la musique est cruciale, le spectaculaire ne l'est pas moins et sur deux plans différents, puisque non seulement le visuel est essentiel sur scène, mais également dans les représentations figurées qui en sont données par la suite.

Cendrillon est en effet un prétexte commode à l'exhibition d'un univers doublement illusionniste au théâtre puisqu'il s'agit de représenter un monde onirique ${ }^{21}$. Tous les effets scéniques utilisés par ailleurs couramment sont donc convoqués pour créer l'enchantement, avec une insistance accumulative censée créer ce supplément de magie que suggère le conte. Les ballets usent et abusent ainsi des cortèges de personnages improbables ${ }^{22}$, réunissant le maximum de danseurs et de figurants sur le plateau au final, mais les féeries ne sont pas en reste avec leur utilisation paroxystique de l'esthétique du tableau, voire de l'« apothéose », et le phénomène culmine, en ce qui concerne Cendrillon, dans la version de 1866, avec une distribution qui adjoint aux vingt-trois rôles nommés les

Princesses des Îles des Volcans, des Îles Bleues, des îles dansantes, des grottes de cristal, des îles des Fleurs et des Papillons, Princesses de Trébizonde, des Îles sauvages, etc. seigneurs et dames de la cour, Pages, Valets, Centaures, Gardes de toutes sortes, Cour d'amour, Fées, les Gens de la noce, Villageois, Paysannes, Monstres, Génies du feu, Vers luisants, etc.

La magie nécessite cependant d'autres ressources pour paraitre « réelle ${ }^{23}$ ». Le ballet invente la torture des pointes pour, paradoxalement, suggérer l'évanescence ${ }^{24}$; les autres genres multiplient changements de décor et de costume à vue pour donner un lustre inouï aux spectacles féeriques. Au mépris souvent de la littérature, en particulier 
dans les parodies d'Étienne, qui s'en moquent non sur le plan textuel, mais scénographique ${ }^{25}$. Ainsi, dans La Chatte merveilleuse, pour ironiser sur la rose d'Étienne (symbole transparent de ce qui donne de l'esprit aux filles ${ }^{26}$ ), la marmite de l'héroïne se transforme en rosier dans l'âtre: «Ah! ben, v'là qu'est un peu trop fort! ... mon pot-au-feu changé en pot-aux-roses! » (sc. 5). Le talisman offert par Alidor à Cendrillon et qui opérait un changement magique de son intelligence lorsqu'elle la portait à son sein se voit donc grossi aux dimensions d'un trucage élaboré qui dénonce l'invraisemblance $\mathrm{du}$ premier. Et les parodies suivantes de souligner méta-théâtralement le procédé, comme La Fête de Perrault :

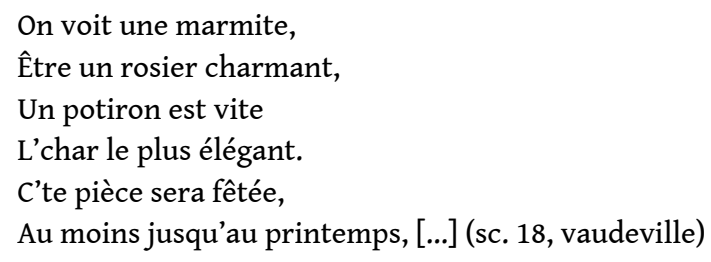

La part du trucage et de ses effets visuels prend même tellement de force et d'importance qu'elle en vient à occulter toute autre considération, et rejaillit sur la compréhension de l'intrigue originale, qui en est sensiblement modifiée. Or ces spectacles sont prolongés par toute une série de "produits dérivés " qui respectent davantage la version scénique que le conte qui en est le prétexte. Selon Annie Renonciat, qui étudie l'imagerie populaire ${ }^{27}$, le phénomène date de 1810 , avec La Chatte merveilleuse, à cause de la publication de planches dont l'une représente la calèche de Cendrillon et de la fée ${ }^{28}$, et il s'est poursuivi par la réalisation de nombreuses œuvres (illustrations, théâtre de papier, etc.) prenant pour sujet les aventures de Cendrillon, en particulier dans l'imagerie d'Épinal. Si bien que, progressivement, le canevas d'origine a subi des altérations qui ont transformé l'histoire dans les mémoires ${ }^{29}$ et qu'il y aurait donc finalement eu « perturbation » de l'intrigue originelle ${ }^{30}$.

On peut donc penser que les perversions narratives fin de siècle doivent beaucoup à la lassitude des nouveaux modèles dramatiques qui avaient fait le succès des théâtres jusqu'à la fin du Second Empire, et qu'elles tentaient ainsi de désamorcer l'aura outrancièrement magique de ceux-ci par une ironie mordante correspondant à un changement d'esthétique radical.

\section{Le « mécano du conte » : l'apothéose du truquiste}

Outrance et surenchère sont en effet les maîtres-mots des spectacles féeriques. Trucs et trucages atteignent des sommets au cours du siècle: l'évolution - tous genres confondus - est extrêmement visible entre 1806 et l'invention du cinéma. Au point que le talent des auteurs finit par s'effacer derrière le génie des décorateurs et des truquistes - et qu'ils se partagent et l'affiche et les recettes.

L'illusion de magie ne requiert pourtant pas toujours des effets très élaborés ou techniquement difficiles à mettre en œuvre : il suffit qu'un personnage identifié par les spectateurs comme une fée fasse mine d'exercer ses pouvoirs pour créer l'attente d'un événement merveilleux. Tout peut alors arriver, et la plus petite modification de l'ordre habituel des choses crée un événement qui passe pour magique. Ainsi, dans La Chatte merveilleuse, le mouvement s'arrête ou la parole se coupe « féeriquement » parce que la fée étend sa baguette : 
MAGDELON, voulant chanter.

Quoi! ...

(Elle n'en peut pas dire davantage.)

[...]

MAGDELON, essayant encore.

Quoi !...

(Même jeu que plus haut. On rit.)

[...]

(La fée étend sa baguette vers Javotte, qui lève la jambe, et donne un coup de pied à

Monsieur de la Canardière, au moment où il se retourne pour faire admirer à

Mirliflor le pied de sa fille.)

$[\ldots]$

MONSIEUR DE LA CANARDIÈRE.

Mais c'est donc le diable qui s'en mêle?

(Il lève les deux bras dans son mouvement de surprise, et reste les deux bras en l'air.)

En vérité, les bras m'en tombent ! (sc. 15)

Ce genre d'effets « faciles », qui consistent à supposer une difficulté là où il n'y en a pas fonctionne tout au long du XIX $x^{e}$ siècle $^{31}$. Bien interprétés, au théâtre ou dans un ballet, ils manifestent inévitablement la magie sur la scène alors qu'il y a là que de banals actes de parole dévoyés et mensongers ou des gestes suggestifs.

De même, on voit au xIX ${ }^{e}$ siècle la persistance et la reprise de trucages vieux d'un siècle et demi : changements de lieu (mais maintenant à vue) justifiés par l'intervention de la puissance tutélaire, apparition de tables bien garnies ou descente de lustres allumés grâce aux trappes et aux cintres, apparition finale de voitures et autres moyens de locomotion fantasmagoriques ${ }^{32}$, etc. Seules la rapidité et la fluidité de ces procédés ont pu progresser, mais le principe de base est toujours le même depuis l'introduction des « feintes » et « secrets » des décorateurs italiens de la Renaissance.

Des nouveautés techniques apparaissent cependant au milieu du siècle : la Cendrillon de 1838 est à cet égard un tournant. Dans ce "vaudeville-féerie en trois journées », la rupture des unités classiques est définitivement consommée au profit du spectaculaire. Non seulement la mise en scène emploie des «transparents ${ }^{33}$ " pour faire voir des apparitions de personnages imaginaires censés être magiquement évoqués, mais c'est la première fois dans le corpus des Cendrillon qu'une apothéose est notée.

Ainsi, à partir de 1838, la complexification de l'intrigue va très nettement de pair avec la multiplication des tableaux et des trucages, jusqu'à atteindre la boursouflure de la grande féerie de 1866 qui exhibe fièrement trente tableaux, trois auteurs et trois décorateurs, plus un truquiste ${ }^{34}$. Ses cinq actes transportent le spectateur, de changement à vue en changement à vue, du manoir de La Pinchonnière (père de Fleurette-Cendrillon) aux serres du roi Hurluberlu xIx, de la montagne du génie du feu Farhulaz à la grotte de Luciole, fée des vers luisants. Et l'intrigue se complique d'épisodes farcesques et de scènes nocturnes, de l'enlèvement de l'héroïne par Farhulaz, et d'une cour d'Amour, péripéties propices à rehausser le spectaculaire et à voir des femmes légèrement vêtues (Charmant était interprété par $\mathrm{M}^{\mathrm{me}}$ Desclozas). Le tout à grand renfort de lumière électrique ${ }^{35}$, d'apothéoses (à chaque fin d'acte) et de « clous».

Certaines scènes reprennent encore le modèle d'Étienne et utilisent la musique de Nicolo, comme lorsque la fée étend sa baguette et que l'air d'opéra entonné par $\mathrm{M}^{\mathrm{me}} \mathrm{de}$ La Houspignole se transforme en chansonnette égrillarde (acte II, tableau 2, sc. 3). Mais 
au contraire de l'opéra-féerie de 1810, le personnage de la fée est essentiel. La fée Luciole est en effet très employée, pour des changements à vue de type traditionnel :

(Elle [la fée] se métamorphose en jeune fée; et l'escalier de marbre se transforme en char ailé qui l'emporte dans les airs. Changement à vue.) ( ( ${ }^{\text {er }}$ acte, tableau 2, scène $\mathrm{V}$ )

comme pour des effets d'une complexité ahurissante :

(Au commandement de la fée, on voit tous les meubles grimper le long des murs jusqu'au plafond. La grande armoire, la table de nuit, les chaises, le coucou et le lit de Riquiqui virent et se renversent. À ce moment, la chambre aussi se met à l'envers. Le plafond devient le plancher, le plancher le plafond. Les fenêtres, les portes et les tableaux, se retournent. Jolicoco, la tête en bas, sort du coucou et crie comme un possédé. Riquiqui s'agite, épouvanté, dans son lit, les bras pendants. Grand brouhaha. - Changement à vue.) (III, 1, V)

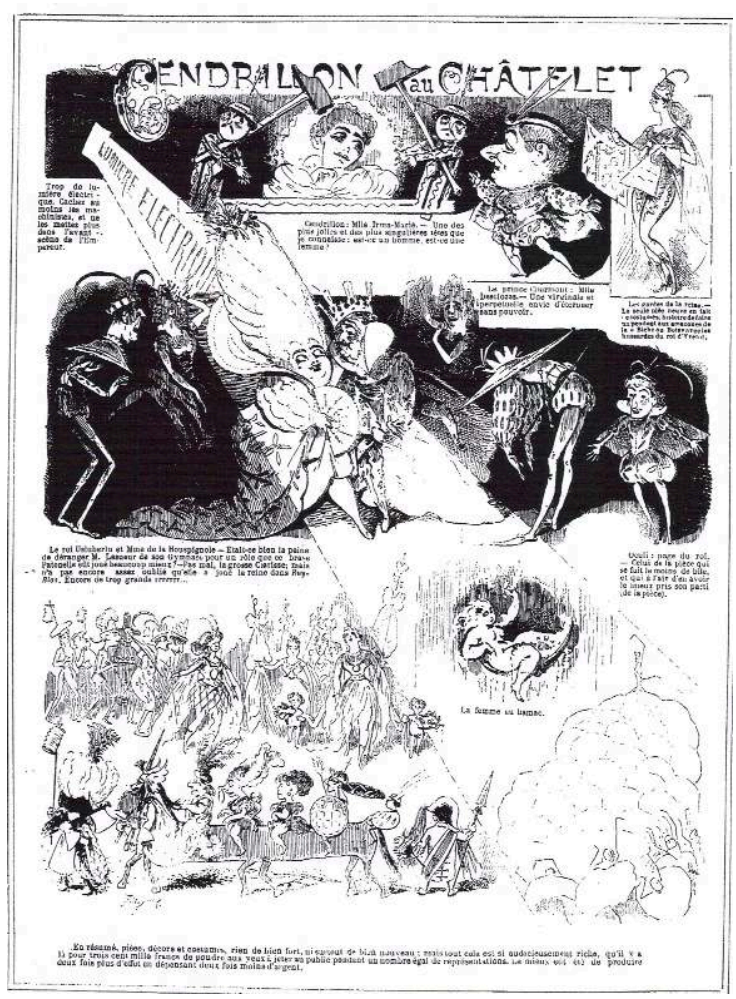

La Vie parisienne, 16 juin 1866 (collection Arch'Yves)

L'outrance de la pièce apparut exceptionnelle, même aux yeux de ses contemporains, et elle fut plusieurs fois caricaturée (voir également un dessin du 10 juin 1866 par Gill, dans La Lune, consultable sur le site de la BnF).

34 À ce compte, il paraît normal que ce soit elle qui conclue la pièce :

([...] Le théâtre devient obscur; quand le jour renaît, commence l'apothéose de M. Fromont, divisée en quatre parties. Pendant la dernière phase, on voit la Fée des vers luisants unissant le prince Charmant à Cendrillon.) (dernière didascalie du texte)

C'est pourtant à Charmant que revient la charge de tirer la morale de l'histoire, pourvu qu'on impute sa réplique à la féerie dramatique :

\section{Charmant}

[...] À quoi me servirait d'être prince, s'il fallait justifier mes caprices; si je ne pouvais en avoir que d'ordinaires, si j'étais obligé de rendre possibles les ordres que je donne? (IV, 2, I) 
comprend sans peine que ce genre de spectacles ait essuyé les mêmes critiques que les opéras au $\mathrm{XVII}^{\mathrm{e}}$ siècle: trop de bruit de machines et un temps de latence exagérément long entre les changements, inanité de l'intrigue ${ }^{36}$ et du texte en général ${ }^{37}, \ldots$ Si bien que, pour stigmatiser la disparition de la littérature derrière la technique, le 18 novembre 1866, La Lune présente une vignette de Gill (dans une demi-page qui illustre le programme des théâtres) représentant un vulgaire godillot, agrémentée d'une légende lapidaire: «Ci-jointe la pantoufle du seul artiste de la maison, M. le machiniste ».

avatar burlesque de la pantoufle de Cendrillon, le soulier de M. Riotton a clos après les reprises de 1879 à la Porte Saint-Martin, et de 1888, au Châtelet - la vogue théâtrale des Cendrillon à grand spectacle ${ }^{38}$. Il n'a cependant pas tari totalement cette mode non plus que l'attrait de la magie puisque, pour l'une de ses premières œuvres importantes - $120 \mathrm{~m}$ de pellicule pour 6 minutes de film - Méliès est parvenu à évoquer parfaitement les pouvoirs de la fée grâce au «fondu » et au montage, et que celle de Prokofiev (1940-1944, sur un livret de Volkov) continue de nous enchanter, dans sa version originale ou celle de 1986, chorégraphiée par Nouréjev ${ }^{39}$. Après tout,

Une fée marraine, il faut que ça serve

Un soir de bal à l'opéra !40

\section{NOTES}

1. Voir les autres articles de ce recueil.

2. Voir les divertissements organisés à Sceaux, par exemple.

3. Voir Jean de Palacio, Les Perversions du merveilleux - Ma Mère l'Oye au tournant du siècle, Séguier, 1993.

4. Ce genre, peu connu voire négligé, vient de faire l'objet d'une ample réhabilitation par Roxane Martin, dans sa thèse: La Féerie romantique sur les scènes parisiennes (1791-1864), Paris, Champion, 2007. Il va sans dire que cet article lui doit énormément pour tout ce qui concerne les cadres théoriques et l'évolution historique.

Que soit ici également remerciée Sylviane Robardey pour son aide amicale si précieuse.

5. Théophile Gautier conclut : «Le Sylphe d'or, la Fille du Danube, la Sylphide, la Fille de l'air, le Lac des fées, Peau-d'Âne, les Pilules du diable, c'est toujours la même chose, un mauvais génie qui dispose des pétards, de l'esprit-de-vin et des trappes, une fée bienfaisante à qui reviennent de droit les nuages de carton, les soleils de fer-blanc, les guirlandes de fleurs en papier et les feux de Bengale de l'apothéose » (La Presse, 6 mai 1839, cité par R.Martin, ouvr. cité. La Sylphide, la Fille du Danube sont des ballets ; le Lac des fées un opéra ; le Sylphe d'or, la Fille de l'air et les Pilules du diable, des féeries).

6. Et jusqu'à nos jours, puisque le plus récent avatar de l'histoire de Cendrillon est l'opéra pour enfant de Peter Maxwell Davies (1980) produit en 1986 à l'Opéra-comique dans son adaptation en 
français de François Sauvageot. La production des Cendrillon a cependant sensiblement fléchi au $\mathrm{Xx}^{\mathrm{e}}$ siècle.

Pour poursuivre la chronologie, on pourra consulter H.Laplace-Claverie, Modernes Féeries - Le Théâtre du XXe siècle entre réenchantement et désenchantement, Paris, Champion, 2007.

7. Alphonse Leveaux, Nos Théâtres, de 1800 à 1880 : la tragédie, le drame, la comédie, l'opéra français, l'opéra italien, l'opéra-comique, le vaudeville, les ballets, l'opérette, la féerie, les revues, la parodie, la pantomime, Paris, Tresse et Stock éditeurs, 1881-1886, p. 197-198.

8. Les auteurs de parodies ne manquent pas de souligner le fait en brodant sur ce thème. Dans $L a$ Fête de Perrault, le personnage éponyme s'écrie, pour critiquer l'adaptation sur le théatre des Ombres chinoises de Séraphin : «M. Séraphin ne me conduira pas à l'immortalité ; c'est même dire que je suis mort, que de me conduire chez les ombres» (sc. 6). Quant à A. Leveaux, il conclut sur cette constatation : «Quand il fit ses contes ingénieux, si naifs et si spirituels à la fois, comme le Chat botté, une perle, un petit chef-d'œuvre en huit pages, il ne se doutait pas du brillant avenir qui les attendait et était bien loin de croire qu'ils immortaliseraient son nom » (ouvr. cité, p. 200).

9. Au moins cinq Belle au Bois-dormant, quatre Petit Poucet, quatre Petit Chaperon rouge, etc. au XIX siècle. R.Martin compte que, sur une centaine de féeries répertoriées entre 1807 et 1830, un tiers se référait à Perrault (ouvr. cité).

10. On dénombre parmi elles, outre le film de Méliès et dix-neuf pièces à l'appartenance générique floue (féerie, folie, parodie, et leurs composés, pour acteurs, acrobates ou marionnettes), une comédie, quatre ballets et quatre opéra/opéras-comiques/opérette.

On consultera avec intérêt, outre les textes, deux catalogues d'exposition de la Bibliothèque nationale de France consacrés aux contes de fées et à Cendrillon en particulier : Il était une fois... les contes de fées, Seuil-BNF, 2001, et Cendrillon à l'Opéra et quelques autres scènes parisiennes [...], mars-juin 2001. Ainsi qu'en ligne, le dossier sur Cendrillon (http://www.bnf.fr/contes/gros/ cendrill/index.html).

11. "Mélodrame-féerie ", " grand ballet pantomime ", "folie-vaudeville ", « folie-parodie », etc.

12. Cendrillon ou la Petite Pantoufle de verre, mélodrame-féerie à grand spectacle, en 4 actes et en prose, Orné de chants, danses, métamorphoses, costumes analogues au sujet: deux divertissements, le $1^{\mathrm{er}}$ au $2^{\mathrm{e}}$ acte, l'autre à la fin de la pièce. Paroles de MM. Alexandre et Constant, musique de M.Bianchi, ballets de M.Adam, Théâtre des Élèves de la rue de Thionville, 26 mars 1806.

13. Cendrillon, paroles de M.Étienne, musique de M.Nicolo Isouard, opéra-comique, 22 février 1810.

14. Cendrillon ou la Bonne Marraine, grand ballet-pantomime en 3 actes de Jean-Baptiste Blache père, décorations de M.Olivier, costumes de M.Orléans, machines de l'invention de MM. Dauzets (publié à Bordeaux en 1823).

15. Cendrillon, conte de fées en 4 actes et 6 tableaux, livret d'Henri Cain, musique de Jules Massenet, opéra-comique, 24 mai 1899. Un (rare) enregistrement est disponible chez Sony et le livret est consultable sur internet (www.karadar.com/librettos/massenet_cendrillon.html).

16. Selon le concept mis en lumière par Claude Bremond dans Le Magazine littéraire, "Contes et mémoire du peuple », 150, juillet-août 1979, p. 13-16, et redéfini dans la dernière livraison de Féeries (3, 2006, p. 181-213).

17. "Cendrillon sur la scène parisienne : les avatars d'un mythe ou d'une fidélité illusoire ", Il était une fois... les contes de fées, ouvr. cité, p. 263.

18. Voir J.de Palacio (ouvr. cité), qui consacre tout un chapitre à Cendrillon et à ses sœurs narratives.

19. Contrairement à Riquet à la houppe, par exemple, qui ironise légèrement sur l'âge de la princesse à son réveil et le style désormais démodé de sa robe. 
20. Cette pièce doit visiblement beaucoup au XVII ${ }^{\mathrm{e}}$ siècle puisque le monologue de Tisbé (III, 1) parodie un lamento de magicienne d'opéra. La reprise en 1877 au Châtelet insista d'ailleurs lourdement sur ces influences, en insérant des airs anciens dans les scènes de danse et en exhibant des costumes inspirés du Grand Siècle, en particulier celui de la fée, littéralement décalqué sur celui de l'Armide de Quinault (1686) - Voir les maquettes de costumes par Théophile Thomas sous la cote D.216 (O.C.1) à la Bibliothèque de l'Opéra.

21. Il n'y a dans le corpus que peu d'exceptions: Sophie ou la Nouvelle Cendrillon (Théâtre de l'Impératrice, 6 novembre 1810), La Cendrillon des Écoles ou le Tarif des prix (10 novembre 1810), et la Cendrillon de Th. Barrière (Théâtre du Gymnase, 23 décembre 1858), qui sont deux comédies intégralement parlées et une " comédie-vaudeville ». La première, bien qu'elle soit apparue dans le sillage de la vogue des Cendrillon de 1810 reprend la trame du conte sur le mode de la comédie traditionnelle, avec le renfort d'un matelot gascon, d'un jardinier, d'une confidente (Jeannette, la fille du jardinier), d'un jeune premier faisant fonction de secrétaire de la méchante $\mathrm{M}^{\mathrm{me}}$ Delmar, et de M.Delmar enfin, revenant des Amériques, qui occupe le rôle de la fée providentielle en organisant, pour les punir, la mystification de sa femme et ses deux belles-filles; la pièce se termine sur un double mariage et une reconnaissance (M. Delmar apporte une fortune au gentil secrétaire de la part de son père, mort à la Jamaïque) ainsi qu'une large réconciliation familiale (Sophie pardonne ses malheurs et M.Delmar se réconcilie avec sa femme). La deuxième, en un acte, se déroule dans un pensionnat où une orpheline est sciemment mise à l'écart des récompenses au profit de camarades plus fortunées. La troisième enfin, se veut plus sérieuse encore, en jouant la carte de l'apitoiement envers une hérö̈ne jalouse de sa sœur mieux aimée par leur mère. Ces pièces, "réalistes", n'utilisent en fait le prétexte de Cendrillon que par analogie avec leur canevas ou pour de allusions ponctuelles (dans le dernier texte, un domestique lit le conte et laisse traîner le livre pour essayer d'éclairer la mère sur sa préférence éhontée).

22. On trouve des « Gouttes de rosée » dansantes chez Massenet!

23. Comme le remarque M.-F. Christout, plus le merveilleux se veut immatériel, plus il recourt à du matériel (voir "Techniques et effets du merveilleux dans le ballet et les arts voisins ", RHT, 1963, p. 88).

24. L'Opéra avait depuis longtemps déjà trouvé d'autres moyens de faire voler ses chanteuses, à grand renfort de harnais et de poulies, et il va, pour les œuvres de Wagner, construire des chariots à roulettes poussés à la main et cachés derrière des voiles.

25. En ce qui concerne cette tendance à la caricature scénique, voir R.Martin, ouvr. cité.

26. La fée Minette l'explicite dans un couplet : «Tu perdis en fuyant/ Ta chaussure et ta rose... / Fille qui s'oublie un instant, / Perd toujours quelque chose » (sc. 18).

27. «Et l'image, en fin de conte ? Suites, fantaisies et variations sur les contes de Perrault dans l'imagerie », Romantisme - Revue du XIX ${ }^{e}$ siècle, 78, 1992, p. 103-125.

28. Cette image est numérisée et visible sur le site de la BNF déjà mentionné.

29. On se trouve donc face à un phénomène en tout point comparable à la mutation des histoires opérée dans l'esprit des enfants qui ont grandi avec les adaptations de Perrault par Disney.

30. «Un grand nombre d'indices nous prouvent que le succès de l'opéra-féerie a fait écran à celui de Perrault au point qu'une réelle contamination du premier sur le second s'opère dans l'imagerie » (A. Renonciat, art. cité, p. 113).

31. Ces effets sont aussi vieux que les pièces de théâtre d'inspiration magique et fonctionnent encore chez Méliès lorsque Cendrillon, en quête de carrosse, ne peut soulever une citrouille, visiblement trop lourde ( $1^{\text {re }}$ partie, $7^{\mathrm{e}}$ tableau). Et il y a fort à parier qu'ils fonctionneront encore longtemps, tant au cinéma que sur la scène.

32. Dans Le Rendez-vous des Cendrillons, la dernière scène fait défiler toutes les Cendrillons (affublées de noms distinctifs) dans le véhicule qui les caractérise le mieux : « Nicole est dans une voiture faite avec des tambours de basque, et trainée par une cigale.... Blondine, dans une vinaigrette, peinte de diverses couleurs, et traînée par un geai, paré de plumes de paon.... Jocrisse 
est sur un potiron, traîné par deux rats, conduit par une chatte, avec deux souris pour laquais.... Simplette est sur un mouton, dont la tête est entourée de fleurs.... ", sc. 14). On se croirait dans une mascarade baroque ou un opéra classique...

33. " (La porte de la chambre où est entré Mélidor s'éclaire tout à-coup, et l'on voit sur un transparent un chevalier, la lance en arrêt.) » (1 ${ }^{\text {re }}$ journée, sc. 4)

34. Cendrillon ou la Pantoufle merveilleuse, grande féerie en cinq actes et trente tableaux par MM. Clairville, Albert Monnier et Ernest Blum, musique nouvelle de M.Victor Chéri, ballets de M.Honoré, décors de MM. Chéret, Fromont, Robecchi, machines de M.Riotton, costumes dessinés par M.Grevin, représentée pour la première fois, à Paris, sur le Théâtre impérial du Châtelet, le lundi 4 juin 1866, direction de M.Hippolyte Holstein.

35. Le texte insiste à plusieurs reprises sur les rayons de lumière électrique nécessaires (sur les costumes des Vers luisants, sur les pantoufles de verre en particulier).

36. Comme le note R.Martin (ouvr. cité), la « particularité de l'écriture féerique tient dans le fait que, contrairement au mélodrame, le recours aux effets scéniques n'agit pas comme un moyen de nouer l'intrigue et d'entretenir le suspense ; le spectacle est placé au premier plan, et l'intrigue n'intervient que pour assurer la fluidité de l'œuvre ». Les prodiges de la fée électricité sont tellement à la mode au théâtre que le journal La Lune, en date du 28 octobre 1866, publie en première page un dessin de Gill où l'on voit une actrice en maillot baignée d'un flot de lumière courageusement endigué par un machiniste à l'extrême gauche: «[...] Nous avons du carton peint, de la lumière électrique, des trucs et du strass, et du chrysocale, et des couplets idiots, mais indécents, panachés de calembours caducs, etc., etc. Nous avons tout ça, et nous avons encore des maillots roses avec quelque chose dedans, le plus de choses possible dedans.[...]».

37. Les critiques contemporains sont unanimes sur ce point et rivalisent d'esprit caustique pour railler ce travers: "Nous nous sommes demandé plus d'une fois en assistant à cette féerie [L'Étoile du berger] infiniment trop surchargée de musique, pourquoi l'on ne supprimerait pas, dans ces sortes de pièces, les chœurs et le dialogue se contentant de montrer les décorations, les costumes, les cortèges, les trucs et les surprises sans aucun mélange de littérature. Une affiche ainsi conçue : «La pièce a été supprimée comme nuisant aux décorations ", attirerait assurément beaucoup de monde." (Théophile Gautier, Histoire de l'art dramatique, vol. 4, p. 267, paru initialement dans La Presse, 18 mai 1846 et cité par R.Martin, ouvr. cité).

38. À l'exception d'une féerie lyrique anonyme en seulement 6 tableaux, datée du 8 janvier 1897, les quatre Cendrillon suivantes (si la liste en est exhaustive) appartiennent à l'opérette, l'opéra-comique et au cinéma. Deux autres Cendrillon dramatiques ont été relevées au $\mathrm{xx}^{\mathrm{e}}$ siècle, mais l'une pour le Collège français de Demoiselles de Madrid (1913) et l'autre à Gand, pour un Cercle artistique et littéraire (1921).

39. La fée y est actualisée en producteur de cinéma...

40. Jean Anouilh, « Le carrosse inutile », Fables, Paris, La Table ronde, 1963.

\section{RÉSUMÉS}

$\mathrm{Au} \mathrm{XIX}^{\mathrm{e}}$ siècle, la féerie est un phénomène dramatique de très grande ampleur. À travers l'exemple des réécritures de Cendrillon pour le théâtre, l'opéra et le ballet, cet article illustre la diversité des variations sur ce thème (plus de trente versions), l'invasion de toutes les scènes par un certain esprit féerique (typique de son époque) et les efforts mis en œuvre pour satisfaire un 
public avide de divertissements oniriques (le machiniste-truquiste, devenu « mécano » du conte, en est sacré le roi). Intertextualité, parodie, transgénéricité, hybridité, ironie et grossissement jusqu'à la boursouflure concourent à réactualiser et remettre à la mode le conte de Perrault, en faisant ainsi un produit caractéristique de la dramaturgie du XIX ${ }^{\mathrm{e}}$ siècle.

\section{Cinderella put into pieces or the second immortality of Perrault in the XIX ${ }^{\text {th }}$ century}

In XIX $^{\text {th }}$ century France, fairy tales are a major theatrical phenomenon. Focusing on the example of rewritings of Cinderella for the stage, this paper illustrates the diversity of variations on this theme (more than thirty versions), the pervasive influence of fantasy and fairy tales on contemporary theatre and the efforts made to satisfy an audience expecting dream-like entertainments (the scene-shifter and maker of special effects becomes the king of this fairyland). Intertextuality, parody, transgenericity, hybridity, irony and magnification to the point of exaggeration contribute to the updating of this fairytale by Perrault and therefore make it a characteristic product of $\mathrm{XIX}^{\text {th }}$ century dramaturgy.

\section{AUTEUR}

\section{NOÉMIE COURTÈS}

Université de Marne-la-Vallée 